\title{
Nanophotonics for Molecular Diagnostics and Therapy Applications
}

\author{
João Conde, ${ }^{1,2}$ João Rosa, ${ }^{1,3}$ João C. Lima, ${ }^{3}$ and Pedro V. Baptista ${ }^{1}$ \\ ${ }^{1}$ CIGMH, Departamento de Ciências da Vida, Faculdade de Ciências e Tecnologia, Universidade Nova de Lisboa, Campus de Caparica, \\ 2829-516 Caparica, Portugal \\ ${ }^{2}$ Instituto de Nanociencia de Aragón, Universidad de Zaragoza, Campus Río Ebro, Edifício I+D, Mariano Esquillor s/n, \\ 50018 Zaragoza, Spain \\ ${ }^{3}$ REQUIMTE, Departamento de Química, Faculdade de Ciências e Tecnologia, Universidade Nova de Lisboa, \\ Campus de Caparica, 2829-516 Caparica, Portugal
}

Correspondence should be addressed to Pedro V. Baptista, pmvb@fct.unl.pt

Received 15 June 2011; Accepted 10 July 2011

Academic Editor: Danuta Wrobel

Copyright ( 2012 João Conde et al. This is an open access article distributed under the Creative Commons Attribution License, which permits unrestricted use, distribution, and reproduction in any medium, provided the original work is properly cited.

\begin{abstract}
Light has always fascinated mankind and since the beginning of recorded history it has been both a subject of research and a tool for investigation of other phenomena. Today, with the advent of nanotechnology, the use of light has reached its own dimension where light-matter interactions take place at wavelength and subwavelength scales and where the physical/chemical nature of nanostructures controls the interactions. This is the field of nanophotonics which allows for the exploration and manipulation of light in and around nanostructures, single molecules, and molecular complexes. What is more is the use of nanophotonics in biomolecular interactions - nanobiophotonics - has prompt for a plethora of molecular diagnostics and therapeutics making use of the remarkable nanoscale properties. In this paper, we shall focus on the uses of nanobiophotonics for molecular diagnostics involving specific sequence characterization of nucleic acids and for gene delivery systems of relevance for therapy strategies. The use of nanobiophotonics for the combined diagnostics/therapeutics (theranostics) will also be addressed, with particular focus on those systems enabling the development of safer, more efficient, and specific platforms. Finally, the translation of nanophotonics for theranostics into the clinical setting will be discussed.
\end{abstract}

\section{Introduction}

Nanophotonics deals with the interaction of light with matter at a nanometer scale, providing challenges for fundamental research and opportunities for new technologies, encompassing the study of new optical interactions, materials, fabrication techniques, and architectures, including the exploration of natural and synthetic, or artificially engineered, structures such as photonic crystals, holey fibers, quantum dots, subwavelength structures, and plasmonics $[1,2]$. The use of photonic nanotechnologies in medicine is a rapidly emerging and potentially powerful approach for disease protection, detection, and treatment. The high speed of light manipulation and the remote nature of optical methods suggest that light may successfully connect diagnostics, treatment, and even the guidance of the treatment in one theranostic procedure combination of therapeutics with diagnostics (including patient prescreening and therapy monitoring).

Limitations in medical practice are closely associated with the fact that diagnostics, therapy, and therapy guidance are three discrete and isolated stages. In order to overcome some of the sensitivity and specificity of current medicines, theranostics unites the three above stages in one single process, supporting early-stage diagnosis and treatment [3, 4]. Nowadays, there is an ever increasing need to enhance the capability of theranostics procedures where nanophotonicsbased sensors may provide for the simultaneous detection of several gene-associated conditions and nanodevices utilizing light-guided and light-activated therapy with the ability to monitor real-time drug action (see Figure 1). 


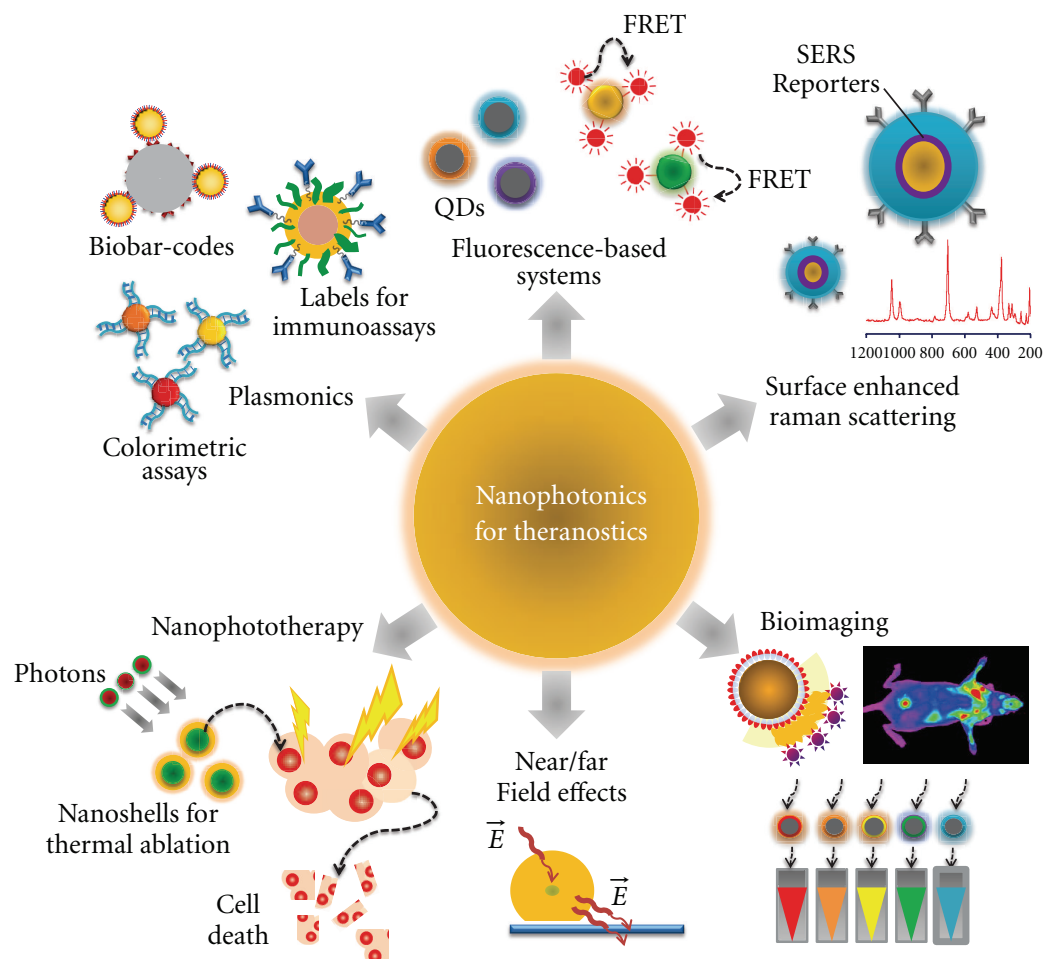

FIGURE 1: Nanophotonics for theranostics. Nanoparticles-based strategies can be used for biosensing using plasmonic nanosensors, such as metal nanoparticles functionalized with nucleic acid strand for colorimetric assays and biobar codes for protein detection or intense labels for immunoassays. Some nanoparticle systems can also be used for sensing by exploring a typical FRET system or can be surrounded with Raman reporters in order to provide in vivo detection and tumour targeting. In fact, NPs symbolize an important class of materials with unique features suitable for biomedical imaging applications such as increased sensitivity in detection and high quantum yields for fluorescence. Alternatively, NPs can survey near/far field enhancing qualities that hold promise for a bounty of novel applications in optics and photonics. Engineered NPs can also act as phototherapeutic agents that can be attached to specific targets for selective damage to cancer cells.

\section{Nanophotonics For Diagnostics}

2.1. Surface Plasmons on Nanoparticles and Surfaces. Surface plasmons are collective charge oscillations that occur at the interface between conductors and dielectrics. They can take various forms, ranging from freely propagating electron density waves along metal surfaces to localized electron oscillations on metal nanoparticles (NPs) [5, 6]. When light passes through a metal nanoparticle, it induces dipole moments that oscillate at the respective frequency of the incident wave, consequently dispersing secondary radiation in all directions. This collective oscillation of the free conduction electrons is called localized surface plasmon resonance (LSPR). Light on NP induces the conduction electrons to oscillate collectively with a resonant frequency that depends on the nanoparticles' size, shape, composition, interparticle distance, and environment (dielectric properties) [7-10]. As a result of these SPR modes, the nanoparticles absorb and scatter light so intensely that single NPs are easily observed by eye using dark-field (optical scattering) microscopy. Plasmonic NPs provide a nearly unlimited photon resource for observing molecular binding for longer periods of time, once they do not blink or bleach like fluorophores [11].

Nanoparticle-based colorimetric assays for diagnostics have been a subject of intensive research, where LSPR can be used to detect DNA or proteins by the changes in the local index of refraction upon adsorption of the target molecule to the metal surface. Due to the intense SPR in the visible yielding extremely bright colors, gold nanoparticle colloids have been widely used of molecular diagnostics. In fact, gold nanoparticles (AuNPs) functionalized with ssDNA capable of specifically hybridizing to a complementary target for the detection of specific nucleic acid sequences in biological samples have been extensively used [12-27]. Other approaches use the AuNPs' plasmonic as a core/seed that can be tailored with a wide variety of surface functionalities to provide highly selective nanoprobes for diagnostics [28] or the SPR scattering imaging or SPR absorption spectroscopy generated from antibody-conjugated AuNPs in molecular biosensor techniques for the diagnosis of oral epithelial living cancer cells in vivo and in vitro [29] and the use of multifunctional AuNPs which incorporate both cytosolic delivery and targeting moieties on the same particle functioning as intracellular sensors to monitor actin rearrangement in live fibroblasts [30].

Plasmonic NPs have also been used as extremely intense labels for immunoassays [31-34] and biochemical sensors $[19,35-37]$. Also, the use of colloidal silver plasmon resonant particles (PRPs) coated with standard ligands as targetspecific labels has been reported for in situ hybridization and 
immunocytology assays [34]. Most notably, a nanoparticlebased Biobar code has been developed for the detection of proteins that relies on magnetic microparticle probes with antibodies that specifically bind a target of interest and nanoparticle probes that are encoded with DNA that is unique to the protein target of interest and antibodies that can sandwich the target captured by the microparticle probes [33]. Haes and coworkers have reported on an optical biosensor based on localized surface plasmon resonance spectroscopy developed to monitor the interaction between the antigen, amyloid- $\beta$-derived diffusible ligands (ADDLs), and specific anti-ADDL antibodies, used in the detection of a biomarker for Alzheimer's Disease [35].

2.2. Raman-Spectroscopy-Based Systems. When light interacts with a substance, it can be absorbed, transmitted, or scattered. Scattered radiation can result from an elastic collision (Rayleigh scattering) or inelastic (Raman scattering). Raman spectroscopy is based on a change of frequency when light is inelastically scattered by molecules or atoms resulting in a molecular fingerprint information on molecular structure or intermolecular interaction of a specific process or molecule. The potential of Raman spectroscopy as biomedical diagnostics tool is rather low due to its low crosssection $\left(\sim 10^{-30} \mathrm{~cm}^{2}\right)$ that results in low sensitivity [38]. However, in 1977, two groups independently described the use of noble metal surfaces to enhance the Raman scattering signal of target molecules [39, 40]-Surface enhancement raman spectroscopy (SERS). Jeanmaire and Van Duyne proposed a twofold electromagnetic field enhancement that was later associated with the interaction between the incident and scattered photons with the nanostructure's LSPR [41]. Simultaneously, Albrecht and Creighton suggested the source of the enhancement to be caused by a specific interaction between an adsorbate and the nanoparticle surface, briefly, a charge transfer from the adsorbate into the empty energetic levels on the metal surface or from the occupied levels of the nanoparticle's surface to the adsorbate [42-44].

Generally, SERS requires that the biological analyte reaches a suitable surface where the substrates are treated as two-dimensional macroscopic surfaces onto which adsorbed molecules suffer a local-field enhancement. Despite direct adsorption not being a good solution because of its dependence on the affinity between substrate and analyte, a method to identify and distinguish different strains of virus based on signal differences generated by the surface aminoacids using silver nanorods has been successfully developed [45]. Using a similar approach of direct adsorption, Pînzary et al. used naked silver nanoparticles to differentiate in situ healthy colon from carcinoma colon tissue [46]. Nanotags have been widely employed to address the lack of specificity $[47,48]$. These nanotags usually possess a metallic colloidal core functionalized with a Raman reporting molecule and the specific molecule used to capture the analyte and have been used to directly detect DNA sequences $[49,50]$ and amplified DNA products of epizootic pathogens using complementary DNA strands so that only the complementary target hybridizes with the probes [51]. Using a similar system, but exploring the distance-dependent enhancement of the electromagnetic field with a hairpin probe molecule, Wabuyele has also been used to distinguish single nucleotide polymorphisms in cancer-related genes [52]. Combining nanotags with other nanoparticles or binding surfaces that target the same analyte in a sandwich conformation proved useful to detect antibodies in serum [53]. A similar approach using a flat substrate instead of NPs had already been proposed to detect DNA, RNA, and proteins [54, 55]. However, in this approach, the substrate is used only to immobilize the analyte; a gold-nanoparticle-based nanotag is used to identify the analyte and the surface enhancement is obtained by silver coating of the nanotag. miRNA profiling has also been pursued via a slightly different approach based on the hybridization of the target molecules with a thiolated oligonucleotide and subsequent functionalization on a silver substrate [56]. SERS have also been explored to identify changes in the analyzed system such as interaction between DNA and xenobiotic molecules like cisplatin [57] or DNAbinding proteins $[58,59]$. The combination of magnetic iron/gold core-shell nanoparticles with gold nanorods has also been used to specifically enumerate $E$. coli in water samples in a rapid and sensitive test [60]. In this case, the magnetic nanoparticles are used to concentrate the bacteria, improving the Raman signal by concentration and the posteriorly added gold nanorods serve as Raman signal enhancers.

SERS can also be used in conjunction with colloidal gold to detect and target tumors in vivo, where the AuNPs are surrounded with Raman reporters that provide light emission 200 times brighter than quantum dots [61, 62]. It was also found that the Raman reporters became more stable and yielded larger optical enhancements when NPs were encapsulated with a thiol-modified polyethylene glycol coat, which also allows for increased biocompatibility and circulation times in vivo. When conjugated to tumortargeting ligands, these conjugated SERS-NPs were able to target tumor markers at surface of malignant cells, such as epidermal growth factor receptor (EGFR) that is sometimes overexpressed in cells of certain cancer types [29] and used to locate the tumor in xenograft tumor models [50].

2.3. Fluorescence-Based Systems. Quantum dots (QDs) are semiconductor nanoparticles with narrow, tunable, symmetrical emission spectra, and high quantum yields [63-65], and together with compatibility with DNA and proteins, make QDs exceptional substitutes as fluorescence labels. The use of QDs for nucleic acid characterization has long been proposed, for example, CdSe/ZnS QDs for SNP identification on human TP53 gene, multiallele detection of hepatitis B and C viruses [66], and in situ detection of chromosome abnormalities and mutations [67]. QDs have also been used as chemical sensors by exploring a typical FRET system where a dark quencher is placed at a proteinbinding site attached to a QD surface. The quantum dots emission is quenched in presence of the analyte and upon analyte displacement the emission is restored [68]. A simpler approach was used to detect adenine using fluorescent $\mathrm{ZnS}$ nanoparticles at $\mathrm{pH} 7$, making use of capability of 
adenine itself to quench emission of the quantum-dot-like nanoparticles [69].

Several studies report on the modulation of fluorophores at the vicinity of nanoparticles (e.g., gold, silver, and quantum dots) [70, 71], an interaction that has found application in a variety of systems to detect biologically relevant targets with particular focus upon AuNPs due to their ease in functionalization with biomolecules [72-75]. Several methods based on the quenching of fluorescence have been proposed for DNA detection consisting of fluorophore-labeled ssDNA electrostatically adsorbed onto gold nanoparticles [76], carbon nanotubes [77], and carbon nanoclots [78], where the presence of a complementary target triggers desadsorption of the newly formed dsDNA from the nanostructures due to the electrostatic variation between ssDNA and dsDNA, and fluorescence emission is restored. Also, fluorescence quenching of fluorophores close to metal nanoparticles functionalized with thiol-modified oligonucleotides has been explored in different conformations. Tang and co-workers proposed a method to probe hydroxyl radicals using an AuNP-oligonucleotide-FAM system where the hydroxyl radical promotes strand breakage and consequent release of FAM, restoring the previously quenched fluorescence [79]. The same quenching mechanism was used to detect specific DNA strands using two probes (one with an AuNP label and another labeled with TAMRA) that hybridize to two DNA sequences near each other [80], bringing the fluorophore and AuNP close enough to quench fluorescence emission.

Proteins have also been probed through nanoparticlefluorescence-mediated systems, for example, human blood proteins have been let to interact with fluorescent AuNPs and detected through quenching [81]. In another example, a sandwich immunoassay using AuNPs quenching has been proposed for the detection of the protein cardiac troponin $\mathrm{T}$ by its interaction with two different antibodies, one attached to AuNPs and the other labeled with fluorescent dyes [82]. By means of an opposite modulation, infrared fluorescent nanoparticles showed enhanced fluorescence when interacting with protein [83].

A popular application of fluorescence modulation by nanoparticles has been specific ion sensing. Su and coworkers developed a copper sensor by covering fluorescent DNA-Cu/Ag nanoclusters with mercaptopropionic acid which quenches the intrinsic fluorescence of the nanoparticle; in the presence of $\mathrm{Cu}^{2+}$, the capping agent is oxidized to form a disulfide compound resulting in release of the nanoparticle and restoration of emission suitable for quantification between 5 and $200 \mathrm{nM}$ [84]. A very specific colorimetric and fluorimetric method to detect $\mathrm{Hg}^{2+}$ ions was developed with porphyrin-modified $\mathrm{Au} @ \mathrm{SiO}_{2}$ nanoparticles, where the intensively fluorescent red complex turns green and weakly fluorescent in presence of $\mathrm{Hg}^{2+}$ [85]. Another examples include sensing of $\mathrm{Pb}^{2+}$ and adenosine by combining an adenosine aptamer and a DNAzyme with an abasic site where 2-amino-5,6,7-trimethyl-1,8naphthyridine is trapped to quench its fluorescence [86]. When in solution, $\mathrm{Pb}^{2+}$ enables the DNAzyme to cleave its substrate thus removing the fluorescent compound from the abasic site restoring its fluorescence. Similarly, the presence of adenosine induces structural change of the aptamer, resulting in the release of the fluorescent molecule from the DNA duplex and a subsequent fluorescence enhancement.

2.4. Nanophotonics Bioimaging. Nanoparticles show unique features suitable for biomedical imaging applications, such as an increased sensitivity in detection through amplification of signal changes (e.g., magnetic resonance imaging); high fluorescence quantum yields and large magnetic moments; properties that induce phagocytosis and selective uptake by macrophages (e.g., liposomes); physicochemical manipulations of energy (i.e., quantum dots); among others [87]. Because light absorption from biologic tissue components is minimized at near infrared (NIR) wavelengths, most nanoparticles (e.g., noble metal and magnetic NPs, nanoshells, nanoclusters, nanocages, nanorods and quantum dots) for in vivo imaging and therapy have been designed to strongly absorb in the NIR and used for in vivo diagnostics $[83,88,89]$. Ex vivo and in vivo imaging applications of nanoparticles have included their use as contrast agents for magnetic resonance imaging (MRI) [90], optical coherence tomography (OCT) [91-93], photoacoustic imaging (PAI) [94], and two-photon luminescence (TPL) spectroscopy [95].

2.4.1. Magnetic Resonance Imaging. Magnetic resonance imaging (MRI) is based heavily on nuclear magnetic resonance (NMR), first described by R. Damadian. Magnetic resonance measurements cause no obvious deleterious effects on biological tissue, and the incident radiation consists of common radio frequencies at right angles to a static magnetic field [96]. Iron oxide nanoparticles show superparamagnetism, allowing for the facile alignment of the magnetic moments to an applied magnetic field, thus of great interest as contrast agents for MRI [97]. Presently, magnetic iron oxide nanoparticles are routinely used as contrast agents to enhance an MRI image, providing sharper contrast between soft and hard tissue in the body (e.g. liver and spleen or lymph nodes) [98]. Jun et al. presented a synthetically controlled magnetic nanocrystal model system that led to the improvement of high-performance nanocrystal-antibody probe systems for the diagnosis of breast cancer cells via magnetic resonance imaging [99]. Also, $\mathrm{MnFe}_{2} \mathrm{O}_{4}$ nanocrystals functionalized with an antibody conjugate (herceptin) capable of specific targeting of cancerous cells was successfully used for in vivo MRI in mice [88]. Driehuysb et al. developed an imaging method to detect submillimeter-sized metastases with molecular specificity by targeting cancer cells with iron oxide nanoparticles functionalized with cancer-binding ligands, demonstrating in vivo detection of pulmonary micrometastases in mice injected with breast adenocarcinoma cells [100]. Hybrid NPs with a superparamagnetic iron oxide/silica core and a gold nanoshell, with significant absorbance and scattering in the NIR region, have been used in vivo as contrast agents for MRI presenting a good MR signal in hepatoma, each moiety providing for a distinct signal that enhanced detection [101]. 
2.4.2. Optical Coherence Tomography. Optical Coherence Tomography (OCT) is an imaging modality that provides cross-sectional subsurface imaging of biological tissue with micrometer scale resolution which is based on a broadband light source and a fiber-optic interferometer. It captures three-dimensional images from within optical scattering media, typically employing near-infrared light. The use of relatively long wavelength light allows it to penetrate into the scattering medium [102-104]. The extra scattering provided by Au-nanoshells enhances optical contrast and brightness for improved diagnostic imaging of tumors in mice due to the preferential accumulation of the nanoshells in the tumor [105]. Tseng et al. developed nanorings with a localized surface plasmon resonance covering a spectral range of $1300 \mathrm{~nm}$ that produced both photothermal and image contrast enhancement effects in OCT when delivered into pig adipose samples [106]. Additionally, the image contrast enhancement effect could be isolated by continuously scanning the sample with a lower scan frequency, allowing to effectively control the therapeutic modality. In the same way, gold capped nanoroses have been used in photothermal OCT to detect macrophages in ex vivo rabbit arteries [107].

2.4.3. Photoacoustic Imaging. In photoacoustic imaging (PAI) and photoacoustic tomography (PAT), a pulse of NIR laser light, typically $757 \mathrm{~nm}$, is used in resonance with the surface plasmon instead of a continuous NIR source. With this technique causing rapid thermal expansion of the surrounding media, the generated sound wave can be detected on the surface of the subject. NIR reduces the amount of absorption that occurs, but absorption of light by various other organs is unavoidable [108, 109]. Yang et al. demonstrated the feasibility of using poly(ethylene glycol)-coated $\mathrm{Au}$ nanocages as a new in vivo NIR contrastenhancing agent for photoacoustic tomography and image their distribution in the vasculature of rat brain. These Aunanocages enhanced the contrast between blood and the surrounding tissues by up to $81 \%$, achieving a more detailed image of vascular structures at greater depths. Additionally, they were shown to present slight advantages over $\mathrm{Au}$ nanoshells, being better suited for in vivo applications, specially due to their more compact size ( $<50 \mathrm{~nm}$ compared to $>100 \mathrm{~nm}$ for $\mathrm{Au}$-nanoshells) and larger optical absorption cross-sections [110]. Due to the ability of gold-nanorods to have the maximum of the plasmon resonance tuned further into the NIR, Motamedi et al. reported a contrast agent for a laser optoacoustic imaging system for in vivo detection of gold nanorods and to enhance the diagnostic power of optoacoustic imaging [111]. Song et al. proposed a noninvasive in vivo spectroscopic photoacoustic sentinel lymph node mapping in a rat model using gold nanorods as lymph node tracers [112].

2.4.4. Two-Photon Luminescence. In two-photon luminescence (TPL) spectroscopy, an electron is excited from the conductance band to the valence band of the metal nanoparticles using two photons. As the electron relaxes to the conductance band, light is released and amplified due to a resonant coupling with localized surface plasmons, enhancing a variety of linear and nonlinear optical properties $[113,114]$. TPL was first described by Boyd et al. that found that roughened metal surfaces exhibited much higher induced luminescence efficiency than smooth surfaces [115]. In fact, TPL is a potentially powerful technique for noninvasive imaging at the micron scale hundreds of microns deep into scattering tissue. This way, it ought to be possible to discriminate cancerous and healthy tissue based on two-photon imaging from endogenous fluorophores. For enhanced imaging, two-photon contrast agents have been developed showing the ability to increase signal-to-noise ratio and targeted to molecular signatures of interest that are not fluorescent. Because imaging of intrinsic fluorophores is often difficult due to their relatively weak signals, the use of such a bright contrast agent holds the promise to enable in vivo applications of two photon imaging in a clinical setting [113, 116, 117]. Wang et al. collected images of single gold nanorods flowing in the mouse ear blood vessels with luminescence three times stronger than background [114]. It is worth mentioning that the TPL signal from a single nanorod is 58 times that of the two-photon fluorescence signal from a single rhodamine molecule.

2.4.5. QDs for In Vivo Imaging. In the last decade, water soluble bioconjugated QDs have been increasingly applied for imaging $[63,64,118]$ However, QD probes for imaging show poor stability once inside cytosolic environment and reduced biocompatibility in living organisms [119], which constitutes a serious drawback for widespread in vivo application.

Despite the serious concerns related to the in vivo use of QDs, these nanocrystals show remarkable imaging properties that may be judged of value for improved diagnostics. In fact, QDs have proven of great value when imaging vascular networks of mammals such as lymphatic and cardiovascular systems [120-124]. Also, Kim et al. demonstrated that quantum dots allowed a major cancer surgery to be performed in large animals (mice and pigs) under complete image guidance, by locating the position of sentinel lymph nodes [125]. With similar potential to that observed when imaging the lymph system, the imaging of cardiovascular systems has also been achieved using QDs [126, 127]. Larson et al. demonstrated that QDs retained their fluorescence after injection and could be detected in the capillaries of skin and adipose tissue of a mouse [128]. The fluorescent emission and multiplexing capabilities of QDs are being exploited to improve the sensitivity and selectivity in the early detection of tumors [61, 129, 130]. Åkerman et al. described for the first time the application of targeted cancer imaging by using ZnS-capped CdSe QDs coated with a lung-targeting peptide that accumulate in the lungs of mice, whereas two other peptides specifically direct QDs to blood vessels or lymphatic vessels in tumors [131]. Later, Gao et al. described the development of multifunctional nanoparticle probes based on QDs with a copolymer linked to tumor-targeting ligands and drug-delivery functionalities for cancer targeting and imaging in living animals [132]. Once the toxicological aspects associated with QDs have been clarified, such studies 
demonstrate the potential of QDs for ultrasensitive and multiplexed imaging of molecular targets in vivo.

\section{Nanophotonics for Therapy}

Nanophototerapy uses pulsed lasers and absorbing nanoparticles attached to specific targets for selective damage to cancer cells. Plasmonic photothermal therapy (PPTT) and photodynamic therapy (PDT) are two of the main techniques that take advantage of the selective absorbance of the surface plasmon resonance and the fact that the nanoparticles relax by liberating heat into their surrounding environment.

3.1. Plasmonic Photothermal Therapy. Plasmonic photothermal therapy is a less invasive experimental technique that holds great promise for the treatment of cell malignancies and, in particular, of cancer. It combines two key components: (i) light source, specifically lasers with a spectral range of $650-900 \mathrm{~nm}$ for deep tissue penetration and (ii) optical absorption of AuNPs which release the optical irradiation as heat in the picoseconds time scale, thereby inducing photothermal ablation [133-135]. Kirui et al. reported the use of gold and iron oxide hybrid nanoparticles in targeting, imaging, and selective thermal killing of colorectal cancer cells [136]. Huang and colleagues have demonstrated that gold nanorods have a longitudinal absorption band in the NIR on account of SPR oscillations and are effective as photothermal agents [137]. Gold nanorods aspect ratios allow tuning the SPR band from the visible to the NIR (transmits readily through human skin and tissue), making them suitable for photothermal converters of near infrared light for in vivo applications [138, 139]. Effective photothermal destruction of cancer cells and tissue have been demonstrated for other gold nanostructures, such as branched gold nanoparticles [140], gold nanoshells [141143], gold nanocages [134], and gold nanospheres [144].

3.2. Photodynamic Therapy. Photodynamic therapy employs chemical photosensitizers that generate reactive oxygen species (ROS), such as a singlet oxygen $\left({ }^{1} \mathrm{O}_{2}\right)$, capable of tumor destruction $[145,146]$. This technique is noninvasive and can be applied locally or systemically without noticeable cumulative toxicity effects without high costs. To attain maximal killing efficiency of tumor cells, the photosensitizer must be in close proximity to the tumor cells, thus requiring specific targeting when administered systemically. One of the major limitations is the poor tissue penetration of highenergy light and the systemic dispersal of the photosensitizer $[147,148]$.

Aiming at circumventing some of the limitations of photodynamic therapy, Zhang et al. reported a new type of photosensitizers based on photon upconverting nanoparticles (a process where low energy light, usually near-infrared (NIR) or infrared (IR), is converted to higher energies, ultraviolet (UV), or visible, via multiple absorptions or energy transfer steps) $[149,150]$. One year later, Yong et al. reported the use of NPs modified with zinc phthalocyanin photosensitizer that produce green/red emission on near-infrared (NIR) excitation and is capable of singlet oxygen sensitization; upon targeted binding to cancer cells, significant cell destruction was induced [151]. Recently, Qian et al. published similar results with the use of zinc phthalocyanine nanocrystals coated with a uniform layer of mesoporous silica [152].

\section{Conclusions}

Light is an amazing intermediate with a gargantuan capacity for carrying multiple information and functions. Instinctively, we view light as rays, which propagate in a single direction, either being absorbed or reflected to some extent by any object on which it impinges. However, the propagation of light through a material is itself a quantum effect, involving the excitation and relaxation of electrons in the material. It is well known that light has the facility to act through biological, chemical, mechanical, and thermal pathways at molecular/cellular levels in diagnostic and therapeutic applications.

Currently, we are in the dawn of a new age in therapy driven by nanotechnology vehicles. Although there are technical challenges associated with the therapeutic application of nanodevices, the integration of therapy with diagnostic profiling has accelerated the pace of discovery of new nanotechnology methods. In addition to continuing to push forward on the above challenges, nanotechnology together with photonics can be used both for identifying useful target candidates and for validating their importance in disease states. Nanophotonics may present new opportunities for personalized medicine in which diagnosis and treatment are based on each individual's molecular profile. Further research into the fundamental mechanisms that efficiently control light using nanodevices could unveil new dimensions of nanoparticle-mediated theranostic systems.

Here, we have attempted to give the reader a limited overview of some aspects of the current state of research into the fascinating aspects and control over nanophotonics in molecular diagnostics and therapy applications.

\section{Acknowledgment}

The authors acknowledge FCT/MCTES-CIGMH(Portugal) for financial support.

\section{References}

[1] M. Ohtsu, K. Kobayashi, T. Kawazoe, T. Yatsui, and M. Naruse, Principles of Nanophotonics, Series in Optics and Optoelectronics, Taylor \& Francis, CRC Press, 2008.

[2] Y. Shen and P. N. Prasad, "Nanophotonics: a new multidisciplinary frontier," Applied Physics B, vol. 74, no. 7-8, pp. 641645, 2002.

[3] T. Lammers, S. Aime, W. E. Hennink, G. Storm, and F. Kiessling, "Theranostic nanomedicines," Accounts of Chemical Research. In press.

[4] F. Pene, E. Courtine, A. Cariou, and J. P. Mira, "Toward theragnostics," Critical Care Medicine, vol. 37, no. 1, pp. S50-S58, 2009. 
[5] W. L. Barnes, A. Dereux, and T. W. Ebbesen, "Surface plasmon subwavelength optics," Nature, vol. 424, no. 6950, pp. 824-830, 2003.

[6] U. Kreibig and M. Vollmer, Optical Properties of Metal Clusters, vol. 25 of Springer Series in Materials Science, Springer, Berlin, Germany, 1995.

[7] M. C. Daniel and D. Astruc, "Gold nanoparticles: assembly, supramolecular chemistry, quantum-size-related properties, and applications toward biology, catalysis, and nanotechnology," Chemical Reviews, vol. 104, no. 1, pp. 293-346, 2004.

[8] S. Eustis and M. A. El-Sayed, "Why gold nanoparticles are more precious than pretty gold: Noble metal surface plasmon resonance and its enhancement of the radiative and nonradiative properties of nanocrystals of different shapes," Chemical Society Reviews, vol. 35, no. 3, pp. 209-217, 2006.

[9] S. K. Ghosh and T. Pal, "Interparticle coupling effect on the surface plasmon resonance of gold nanoparticles: from theory to applications," Chemical Reviews, vol. 107, no. 11, pp. 4797-4862, 2007.

[10] W. Zhao, M. A. Brook, and Y. Li, "Design of gold nanoparticle-based colorimetric biosensing assays," ChemBioChem, vol. 9, no. 15, pp. 2363-2371, 2008.

[11] J. N. Anker, W. P. Hall, O. Lyandres, N. C. Shah, J. Zhao, and R. P. Van Duyne, "Biosensing with plasmonic nanosensors," Nature Materials, vol. 7, no. 6, pp. 442-453, 2008.

[12] P. Baptista, G. Doria, D. Henriques, E. Pereira, and R. Franco, "Colorimetric detection of eukaryotic gene expression with DNA-derivatized gold nanoparticles," Journal of Biotechnology, vol. 119, no. 2, pp. 111-117, 2005.

[13] P. Baptista, E. Pereira, P. Eaton et al., "Gold nanoparticles for the development of clinical diagnosis methods," Analytical and Bioanalytical Chemistry, vol. 391, no. 3, pp. 943-950, 2008.

[14] Y. C. Cao, R. Jin, C. S. Thaxton, and C. A. Mirkin, "A two-color-change, nanoparticle-based method for DNA detection," Talanta, vol. 67, no. 3, pp. 449-455, 2005.

[15] M. M. C. Cheng, G. Cuda, Y. L. Bunimovich et al., "Nanotechnologies for biomolecular detection and medical diagnostics," Current Opinion in Chemical Biology, vol. 10, no. 1, pp. 11-19, 2006.

[16] J. Conde, J. M. de la Fuente, and P. V. Baptista, "RNA quantification using gold nanoprobes - application to cancer diagnostics," Journal of Nanobiotechnology, vol. 8, article no. $5,2010$.

[17] P. Costa, A. Amaro, A. Botelho, J. Inácio, and P. V. Baptista, "Gold nanoprobe assay for the identification of mycobacteria of the Mycobacterium tuberculosis complex," Clinical Microbiology and Infection, vol. 16, no. 9, pp. 1464-1469, 2010.

[18] G. Doria, R. Franco, and P. Baptista, "Nanodiagnostics: fast colorimetric method for single nucleotide polymorphism/mutation detection," IET Nanobiotechnology, vol. 1, no. 4, pp. 53-57, 2007.

[19] R. Elghanian, J. J. Storhoff, R. C. Mucic, R. L. Letsinger, and C. A. Mirkin, "Selective colorimetric detection of polynucleotides based on the distance-dependent optical properties of gold nanoparticles," Science, vol. 277, no. 5329, pp. 1078-1081, 1997.

[20] H. Li and L. Rothberg, "Colorimetric detection of DNA sequences based on electrostatic interactions with unmodified gold nanoparticles," Proceedings of the National Academy of Sciences of the United States of America, vol. 101, no. 39, pp. 14036-14039, 2004.
[21] C. A. Mirkin, R. L. Letsinger, R. C. Mucic, and J. J. Storhoff, "A DNA-based method for rationally assembling nanoparticles into macroscopic materials," Nature, vol. 382, no. 6592, pp. 607-609, 1996.

[22] W. J. Qin and L. Y. L. Yung, "Nanoparticle-based detection and quantification of DNA with single nucleotide polymorphism (SNP) discrimination selectivity," Nucleic Acids Research, vol. 35, no. 17, article no. e111, 2007.

[23] K. Sato, K. Hosokawa, and M. Maeda, "Rapid aggregation of gold nanoparticles induced by non-cross-linking DNA hybridization," Journal of the American Chemical Society, vol. 125, no. 27, pp. 8102-8103, 2003.

[24] K. Sato, K. Hosokawa, and M. Maeda, "Non-cross-linking gold nanoparticle aggregation as a detection method for single-base substitutions," Nucleic Acids Research, vol. 33, no. 1, article e4, 2005.

[25] J. J. Storhoff, A. D. Lucas, V. Garimella, Y. P. Bao, and U. R. Müller, "Homogeneous detection of unamplified genomic DNA sequences based on colorimetric scatter of gold nanoparticle probes," Nature Biotechnology, vol. 22, no. 7, pp. 883-887, 2004.

[26] T. A. Taton, C. A. Mirkin, and R. L. Letsinger, "Scanometric DNA array detection with nanoparticle probes," Science, vol. 289, no. 5485, pp. 1757-1760, 2000.

[27] C. S. Thaxton, D. G. Georganopoulou, and C. A. Mirkin, "Gold nanoparticle probes for the detection of nucleic acid targets," Clinica Chimica Acta, vol. 363, no. 1-2, pp. 120-126, 2006.

[28] C. C. You, O. R. Miranda, B. Gider et al., "Detection and identification of proteins using nanoparticle-fluorescent polymer 'chemical nose' sensors," Nature Nanotechnology, vol. 2, no. 5, pp. 318-323, 2007.

[29] I. H. El-Sayed, X. Huang, and M. A. El-Sayed, "Surface plasmon resonance scattering and absorption of anti-EGFR antibody conjugated gold nanoparticles in cancer diagnostics: applications in oral cancer," Nano Letters, vol. 5, no. 5, pp. 829-834, 2005.

[30] S. Kumar, N. Harrison, R. Richards-Kortum, and K. Sokolov, "Plasmonic nanosensors for imaging intracellular biomarkers in live cells," Nano Letters, vol. 7, no. 5, pp. 1338-1343, 2007.

[31] W.-C. Law, K.-T. Yong, A. Baev, and P. N. Prasad, "Sensitivity improved surface plasmon resonance biosensor for cancer biomarker detection based on plasmonic enhancement," ACS Nano, vol. 5, no. 6, pp. 4858-4864, 2011.

[32] J. S. Mitchell and T. E. Lowe, "Ultrasensitive detection of testosterone using conjugate linker technology in a nanoparticle-enhanced surface plasmon resonance biosensor," Biosensors and Bioelectronics, vol. 24, no. 7, pp. 2177-2183, 2009.

[33] J. M. Nam, C. S. Thaxton, and C. A. Mirkin, "Nanoparticlebased bio-bar codes for the ultrasensitive detection of proteins," Science, vol. 301, no. 5641, pp. 1884-1886, 2003.

[34] S. Schultz, D. R. Smith, J. J. Mock, and D. A. Schultz, "Singletarget molecule detection with nonbleaching multicolor optical immunolabels," Proceedings of the National Academy of Sciences of the United States of America, vol. 97, no. 3, pp. 996-1001, 2000.

[35] A. J. Haes, L. Chang, W. L. Klein, and R. P. Van Duyne, "Detection of a biomarker for Alzheimer's disease from synthetic and clinical samples using a nanoscale optical biosensor," Journal of the American Chemical Society, vol. 127, no. 7, pp. 2264-2271, 2005. 
[36] A. D. McFarland and R. P. Van Duyne, "Single silver nanoparticles as real-time optical sensors with zeptomole sensitivity," Nano Letters, vol. 3, no. 8, pp. 1057-1062, 2003.

[37] G. Raschke, S. Kowarik, T. Franzl et al., "Biomolecular recognition based on single gold nanoparticle light scattering," Nano Letters, vol. 3, no. 7, pp. 935-938, 2003.

[38] M. K. Hossain and Y. Ozaki, "Surface-enhanced Raman scattering: facts and inline trends," Current Science, vol. 97, no. 2, pp. 192-201, 2009.

[39] D. L. Jeanmaire and R. P. Van Duyne, "Surface Raman spectroelectrochemistry Part I. Heterocyclic, aromatic, and aliphatic amines adsorbed on the anodized silver electrode," Journal of Electroanalytical Chemistry, vol. 84, no. 1, pp. 1-20, 1977.

[40] M. G. Albrecht and J. A. Creighton, "Anomalously intense Raman spectra of pyridine at a silver electrode," Journal of the American Chemical Society, vol. 99, no. 15, pp. 5215-5217, 1977.

[41] B. Pettinger, "Light scattering by adsorbates at Ag particles: quantum-mechanical approach for energy transfer induced interfacial optical processes involving surface plasmons, multipoles, and electron-hole pairs," The Journal of Chemical Physics, vol. 85, no. 12, pp. 7442-7451, 1986.

[42] A. M. Michaels, M. Nirmal, and L. E. Brus, "Surface enhanced Raman spectroscopy of individual rhodamine 6G molecules on large Ag nanocrystals," Journal of the American Chemical Society, vol. 121, no. 43, pp. 9932-9939, 1999.

[43] P. Etchegoin, H. Liem, R. C. Maher et al., "A novel amplification mechanism for surface enhanced Raman scattering," Chemical Physics Letters, vol. 366, no. 1-2, pp. 115-121, 2002.

[44] A. Otto, "On the electronic contribution to single molecule surface enhanced Raman spectroscopy," Indian Journal of Physics, vol. 77B, pp. 63-73, 2003.

[45] S. Shanmukh, L. Jones, J. Driskell, Y. Zhao, R. Dluhy, and R. A. Tripp, "Rapid and sensitive detection of respiratory virus molecular signatures using a silver nanorod array SERS substrate," Nano Letters, vol. 6, no. 11, pp. 2630-2636, 2006.

[46] S. C. Pînzaru, L. M. Andronie, I. Domsa, O. Cozar, and S. Astilean, "Bridging biomolecules with nanoparticles: surface-enhanced Raman scattering from colon carcinoma and normal tissue," Journal of Raman Spectroscopy, vol. 39, no. 3, pp. 331-334, 2008.

[47] S. P. Mulvaney, M. D. Musick, C. D. Keating, and M. J. Natan, "Glass-coated, analyte-tagged nanoparticles: a new tagging system based on detection with surface-enhanced Raman scattering," Langmuir, vol. 19, no. 11, pp. 4784-4790, 2003.

[48] W. E. Doering, M. E. Piotti, M. J. Natan, and R. G. Freeman, "SERS as a foundation for nanoscale, optically detected biological labels," Advanced Materials, vol. 19, no. 20, pp. 3100-3108, 2007.

[49] L. Sun, C. Yu, and J. Irudayaraj, "Surface-enhanced Raman scattering based nonfluorescent probe for multiplex DNA detection," Analytical Chemistry, vol. 79, no. 11, pp. 39813988, 2007.

[50] X. Qian, X. Zhou, and S. Nie, "Surface-enhanced raman nanoparticle beacons based on bioconjugated gold nanocrystals and long range plasmonic coupling," Journal of the American Chemical Society, vol. 130, no. 45, pp. 1493414935, 2008.

[51] K. K. Strelau, A. Brinker, C. Schnee, K. Weber, R. Möller, and J. Popp, "Detection of PCR products amplified from DNA of epizootic pathogens using magnetic nanoparticles and SERS," Journal of Raman Spectroscopy, vol. 42, no. 3, pp. 243-250, 2011.
[52] M. B. Wabuyele, F. Yan, and T. Vo-Dinh, "Plasmonics nanoprobes: detection of single-nucleotide polymorphisms in the breast cancer BRCA1 gene," Analytical and Bioanalytical Chemistry, vol. 398, no. 2, pp. 729-736, 2010.

[53] J. Neng, M. H. Harpster, H. Zhang, J. O. Mecham, W. C. Wilson, and P. A. Johnson, "A versatile SERS-based immunoassay for immunoglobulin detection using antigencoated gold nanoparticles and malachite green-conjugated protein A/G," Biosensors and Bioelectronics, vol. 26, no. 3, pp. 1009-1015, 2010.

[54] Y. C. Cao, R. Jin, and C. A. Mirkin, "Nanoparticles with Raman spectroscopic fingerprints for DNA and RNA detection," Science, vol. 297, no. 5586, pp. 1536-1540, 2002.

[55] Y. C. Cao, R. Jin, J. M. Nam, C. S. Thaxton, and C. A. Mirkin, "Raman dye-labeled nanoparticle probes for proteins," Journal of the American Chemical Society, vol. 125, no. 48, pp. 14676-14677, 2003.

[56] J. D. Driskell and R. A. Tripp, "Label-free SERS detection of microRNA based on affinity for an unmodified silver nanorod array substrate," Chemical Communications, vol. 46, no. 19, pp. 3298-3300, 2010.

[57] A. Barhoumi, D. Zhang, F. Tam, and N. J. Halas, "Surfaceenhanced raman spectroscopy of DNA," Journal of the American Chemical Society, vol. 130, no. 16, pp. 5523-5529, 2008.

[58] D. Graham, R. Stevenson, D. G. Thompson, L. Barrett, C. Dalton, and K. Faulds, "Combining functionalised nanoparticles and SERS for the detection of DNA relating to disease," Faraday Discussions, vol. 149, pp. 291-299, 2011.

[59] C. V. Pagba, S. M. Lane, H. Cho, and S. Wachsmann-Hogiu, "Direct detection of aptamer-thrombin binding via surfaceenhanced Raman spectroscopy," Journal of Biomedical Optics, vol. 15, no. 4, Article ID 047006, 2010.

[60] B. Guven, N. Basaran-Akgul, E. Temur, U. Tamer, and I. H. Boyaci, "SERS-based sandwich immunoassay using antibody coated magnetic nanoparticles for Escherichia coli enumeration," Analyst, vol. 136, no. 4, pp. 740-748, 2011.

[61] W. Cai, D. W. Shin, K. Chen et al., "Peptide-labeled nearinfrared quantum dots for imaging tumor vasculature in living subjects," Nano Letters, vol. 6, no. 4, pp. 669-676, 2006.

[62] J. Kneipp, H. Kneipp, M. McLaughlin, D. Brown, and K. Kneipp, "In vivo molecular probing of cellular compartments with gold nanoparticles and nanoaggregates," Nano Letters, vol. 6, no. 10, pp. 2225-2231, 2006.

[63] W. C. W. Chan and S. Nie, "Quantum dot bioconjugates for ultrasensitive nonisotopic detection," Science, vol. 281, no. 5385, pp. 2016-2018, 1998.

[64] M. Bruchez Jr., M. Moronne, P. Gin, S. Weiss, and A. P. Alivisatos, "Semiconductor nanocrystals as fluorescent biological labels," Science, vol. 281, no. 5385, pp. 2013-2016, 1998.

[65] H. Weller, "Colloidal semiconductor Q-particles: chemistry in the transition region between solid state and molecules," Angewandte Chemie - International Edition, vol. 32, no. 1, pp. 41-53, 1993.

[66] D. Gerion, F. Chen, B. Kannan et al., "Room-temperature single-nucleotide polymorphism and multiallele DNA detection using fluorescent nanocrystals and microarrays," Analytical Chemistry, vol. 75, no. 18, pp. 4766-4772, 2003.

[67] S. Pathak, S. K. Choi, N. Arnheim, and M. E. Thompson, "Hydroxylated quantum dots as luminescent probes for in situ hybridization," Journal of the American Chemical Society, vol. 123, no. 17, pp. 4103-4104, 2001. 
[68] I. L. Medintz, A. R. Clapp, H. Mattoussi, E. R. Goldman, B. Fisher, and J. M. Mauro, "Self-assembled nanoscale biosensors based on quantum dot FRET donors," Nature Materials, vol. 2, no. 9, pp. 630-638, 2003.

[69] L. M. Devi and D. P. S. Negi, "Sensitive and selective detection of adenine using fluorescent ZnS nanoparticles," Nanotechnology, vol. 22, no. 24, Article ID 245502, 2011.

[70] J. Gersten and A. Nitzan, "Spectroscopic properties of molecules interacting with small dielectric particles," The Journal of Chemical Physics, vol. 75, no. 3, pp. 1139-1152, 1981.

[71] K. A. Kang, J. Wang, J. B. Jasinski, and S. Achilefu, "Fluorescence manipulation by gold nanoparticles: from complete quenching to extensive enhancement," Journal of Nanobiotechnology, vol. 9, article 16, 2011.

[72] A. Quarta, R. D. Corato, L. Manna, A. Ragusa, and T. Pellegrino, "Fluorescent-magnetic hybrid nanostructures: preparation, properties, and applications in biology," IEEE Transactions on Nanobioscience, vol. 6, no. 4, pp. 298-308, 2007.

[73] J. R. Lakowicz, J. Malicka, E. Matveeva, I. Gryczynski, and Z. Gryczynski, "Plasmonic technology: novel approach to ultrasensitive immunoassays," Clinical Chemistry, vol. 51, no. 10, pp. 1914-1922, 2005.

[74] J. R. Lakowicz, "Plasmonics in biology and plasmoncontrolled fluorescence," Plasmonics, vol. 1, no. 1, pp. 5-33, 2006.

[75] I. L. Medintz and H. Mattoussi, "Quantum dot-based resonance energy transfer and its growing application in biology," Physical Chemistry Chemical Physics, vol. 11, no. 1, pp. 17-45, 2009.

[76] P. C. Ray, G. K. Darbha, A. Ray, J. Walker, and W. Hardy, "Gold nanoparticle based FRET for DNA detection," Plasmonics, vol. 2, no. 4, pp. 173-183, 2007.

[77] Y. Liu, Y. Wang, J. Jin, H. Wang, R. Yang, and W. Tan, "Fluorescent assay of DNA hybridization with label-free molecular switch: reducing background-signal and improving specificity by using carbon nanotubes," Chemical Communications, no. 6, pp. 665-667, 2009.

[78] W. Bai, H. Zheng, Y. Long, X. Mao, M. Gao, and L. Zhang, "A carbon dots-based fluorescence turn-on method for DNA determination," Analytical Sciences, vol. 27, no. 3, pp. 243246, 2011.

[79] B. Tang, N. Zhang, Z. Chen et al., "Probing hydroxyl radicals and their imaging in living cells by use of FAM-DNA-Au nanoparticles," Chemistry, vol. 14, no. 2, pp. 522-528, 2008.

[80] Z. S. Wu, J. H. Jiang, L. Fu, G. L. Shen, and R. Q. $\mathrm{Yu}$, "Optical detection of DNA hybridization based on fluorescence quenching of tagged oligonucleotide probes by gold nanoparticles," Analytical Biochemistry, vol. 353, no. 1, pp. 22-29, 2006.

[81] S. H. De Paoli Lacerda, J. J. Park, C. Meuse et al., "Interaction of gold nanoparticles with common human blood proteins," ACS Nano, vol. 4, no. 1, pp. 365-379, 2010.

[82] S. Mayilo, M. A. Kloster, M. Wunderlich et al., "Long-range fluorescence quenching by gold nanoparticles in a sandwich immunoassay for cardiac troponin T," Nano Letters, vol. 9, no. 12, pp. 4558-4563, 2009.

[83] X. He, J. Gao, S. S. Gambhir, and Z. Cheng, "Near-infrared fluorescent nanoprobes for cancer molecular imaging: status and challenges," Trends in Molecular Medicine, vol. 16, no. 12, pp. 574-583, 2010.

[84] Y. T. Su, G. Y. Lan, W. Y. Chen, and H. T. Chang, "Detection of copper ions through recovery of the fluorescence of
DNA-templated copper/silver nanoclusters in the presence of mercaptopropionic acid," Analytical Chemistry, vol. 82, no. 20, pp. 8566-8572, 2010.

[85] Y. Cho, S. S. Lee, and J. H. Jung, "Recyclable fluorimetric and colorimetric mercury-specific sensor using porphyrinfunctionalized $\mathrm{Au} @ \mathrm{SiO}_{2}$ core/shell nanoparticles," Analyst, vol. 135, no. 7, pp. 1551-1555, 2010.

[86] Y. Xiang, A. Tong, and Y. Lu, "Abasic site-containing DNAzyme and aptamer for label-free fluorescent detection of $\mathrm{Pb}^{2+}$ and adenosine with high sensitivity, selectivity, and tunable dynamic range," Journal of the American Chemical Society, vol. 131, no. 42, pp. 15352-15357, 2009.

[87] R. Gill, M. Zayats, and I. Willner, "Semiconductor quantum dots for bioanalysis," Angewandte Chemie - International Edition, vol. 47, no. 40, pp. 7602-7625, 2008.

[88] J. H. Lee, Y. M. Huh, Y. W. Jun et al., "Artificially engineered magnetic nanoparticles for ultra-sensitive molecular imaging," Nature Medicine, vol. 13, no. 1, pp. 95-99, 2007.

[89] E. I. Altinoğlu and J. H. Adair, "Near infrared imaging with nanoparticles," Wiley Interdisciplinary Reviews: Nanomedicine and Nanobiotechnology, vol. 2, no. 5, pp. 461-477, 2010.

[90] P. Vartholomeos, M. Fruchard, A. Ferreira, and C. Mavroidis, "MRI-guided nanorobotic systems for therapeutic and diagnostic applications," Annual Review of Biomedical Engineering, vol. 13, pp. 157-184, 2011.

[91] E. V. Zagaynova, M. V. Shirmanova, M. Y. Kirillin et al., "Contrasting properties of gold nanoparticles for optical coherence tomography: phantom, in vivo studies and Monte Carlo simulation," Physics in Medicine and Biology, vol. 53, no. 18, pp. 4995-5009, 2008.

[92] J. C. Kah, M. Olivo, T. H. Chow et al., "Control of optical contrast using gold nanoshells for optical coherence tomography imaging of mouse xenograft tumor model in vivo," Journal of Biomedical Optics, vol. 14, no. 5, Article ID 054015, 2009.

[93] A. L. Oldenburg, M. N. Hansen, T. S. Ralston, A. Wei, and S. A. Boppart, "Imaging gold nanorods in excised human breast carcinoma by spectroscopic optical coherence tomography," Journal of Materials Chemistry, vol. 19, no. 35, pp. 6407-6411, 2009.

[94] X. Yang, E. W. Stein, S. Ashkenazi, and L. V. Wang, "Nanoparticles for photoacoustic imaging," Wiley Interdisciplinary Reviews. Nanomedicine and nanobiotechnology, vol. 1, no. 4, pp. 360-368, 2009.

[95] M. B. Mohamed, V. Volkov, S. Link, and M. A. El-Sayed, "The 'lightning' gold nanorods: fluorescence enhancement of over a million compared to the gold metal," Chemical Physics Letters, vol. 317, no. 6, pp. 517-523, 2000.

[96] R. Damadian, "Tumor detection by nuclear magnetic resonance," Science, vol. 171, no. 3976, pp. 1151-1153, 1971.

[97] D. S. Mathew and R. S. Juang, "An overview of the structure and magnetism of spinel ferrite nanoparticles and their synthesis in microemulsions," Chemical Engineering Journal, vol. 129, no. 1-3, pp. 51-65, 2007.

[98] S. Laurent, D. Forge, M. Port et al., "Magnetic iron oxide nanoparticles: synthesis, stabilization, vectorization, physicochemical characterizations and biological applications," Chemical Reviews, vol. 108, no. 6, pp. 2064-2110, 2008.

[99] Y. W. Jun, Y. M. Huh, J. S. Choi et al., "Nanoscale size effect of magnetic nanocrystals and their utilisation for cancer diagnosis via magnetic resonance imaging," Journal of the American Chemical Society, vol. 127, no. 16, pp. 5732-5733, 2005. 
[100] R. T. Branca, Z. I. Cleveland, B. Fubara et al., "Molecular MRI for sensitive and specific detection of lung metastases," Proceedings of the National Academy of Sciences of the United States of America, vol. 107, no. 8, pp. 3693-3697, 2010.

[101] D. Kim, M. K. Yu, T. S. Lee, J. J. Park, Y. Y. Jeong, and S. Jon, "Amphiphilic polymer-coated hybrid nanoparticles as CT/MRI dual contrast agents," Nanotechnology, vol. 22, no. 15, Article ID 155101, 2011.

[102] D. Huang, E. A. Swanson, C. P. Lin et al., "Optical coherence tomography," Science, vol. 254, no. 5035, pp. 1178-1181, 1991.

[103] J. M. Schmitt, "Optical Coherence Tomography (OCT): a review," IEEE Journal on Selected Topics in Quantum Electronics, vol. 5, no. 4, pp. 1205-1215, 1999.

[104] H. G. Bezerra, M. A. Costa, G. Guagliumi, A. M. Rollins, and D. I. Simon, "Intracoronary optical coherence tomography: a comprehensive review. Clinical and research applications," JACC: Cardiovascular Interventions, vol. 2, no. 11, pp. 10351046, 2009.

[105] A. M. Gobin, M. H. Lee, N. J. Halas, W. D. James, R. A. Drezek, and J. L. West, "Near-infrared resonant nanoshells for combined optical imaging and photothermal cancer therapy," Nano Letters, vol. 7, no. 7, pp. 1929-1934, 2007.

[106] H. Y. Tseng, C. K. Lee, S. Y. Wu et al., "Au nanorings for enhancing absorption and backscattering monitored with optical coherence tomography," Nanotechnology, vol. 21, no. 29, Article ID 295102, 2010.

[107] A. S. Paranjape, R. Kuranov, S. Baranov et al., "Depth resolved photothermal OCT detection of macrophages in tissue using nanorose," Biomedical Optics Express, vol. 1, no. 1, pp. 2-16, 2010.

[108] Y. Su, F. Zhang, K. Xu, J. Yao, and R. K. Wang, "A photoacoustic tomography system for imaging of biological tissues," Journal of Physics D, vol. 38, no. 15, pp. 2640-2644, 2005.

[109] K. S. Valluru, B. K. Chinni, and N. A. Rao, "Photoacoustic imaging: opening new frontiers in medical imaging," Journal of Clinical Imaging Science, vol. 1, article 24, 2011.

[110] X. Yang, S. E. Skrabalak, Z. Y. Li, Y. Xia, and L. V. Wang, "Photoacoustic tomography of a rat cerebral cortex in vivo with Au nanocages as an optical contrast agent," Nano Letters, vol. 7, no. 12, pp. 3798-3802, 2007.

[111] M. Eghtedari, A. Oraevsky, J. A. Copland, N. A. Kotov, A. Conjusteau, and M. Motamedi, "High sensitivity of in vivo detection of gold nanorods using a laser optoacoustic imaging system," Nano Letters, vol. 7, no. 7, pp. 1914-1918, 2007.

[112] K. H. Song, C. Kim, K. Maslov, and L. V. Wang, "Noninvasive in vivo spectroscopic nanorod-contrast photoacoustic mapping of sentinel lymph nodes," European Journal of Radiology, vol. 70, no. 2, pp. 227-231, 2009.

[113] M. B. Mohamed, V. Volkov, S. Link, and M. A. El-Sayed, "The 'lightning' gold nanorods: fluorescence enhancement of over a million compared to the gold metal," Chemical Physics Letters, vol. 317, no. 6, pp. 517-523, 2000.

[114] H. Wang, T. B. Huff, D. A. Zweifel et al., "In vitro and in vivo two-photon luminescence imaging of single gold nanorods," Proceedings of the National Academy of Sciences of the United States of America, vol. 102, no. 44, pp. 15752-15756, 2005.

[115] G. T. Boyd, Z. H. Yu, and Y. R. Shen, "Photoinduced luminescence from the noble metals and its enhancement on roughened surfaces," Physical Review B, vol. 33, no. 12, pp. 7923-7936, 1986.
[116] N. J. Durr, T. Larson, D. K. Smith, B. A. Korgel, K. Sokolov, and A. Ben-Yakar, "Two-photon luminescence imaging of cancer cells using molecularly targeted gold nanorods," Nano Letters, vol. 7, no. 4, pp. 941-945, 2007.

[117] K. Imura, T. Nagahara, and H. Okamoto, "Plasmon mode imaging of single gold nanorods," Journal of the American Chemical Society, vol. 126, no. 40, pp. 12730-12731, 2004.

[118] K. T. Yong, I. Roy, H. Ding, E. J. Bergey, and P. N. Prasad, "Biocompatible near-infrared quantum dots as ultrasensitive probes for long-term in vivo imaging applications," Small, vol. 5, no. 17, pp. 1997-2004, 2009.

[119] A. M. Smith, H. Duan, A. M. Mohs, and S. Nie, "Bioconjugated quantum dots for in vivo molecular and cellular imaging," Advanced Drug Delivery Reviews, vol. 60, no. 11, pp. 1226-1240, 2008.

[120] E. G. Soltesz, S. Kim, R. G. Laurence et al., "Intraoperative sentinel lymph node mapping of the lung using near-infrared fluorescent quantum dots," Annals of Thoracic Surgery, vol. 79, no. 1, pp. 269-277, 2005.

[121] C. P. Parungo, Y. L. Colson, S. W. Kim et al., "Sentinel lymph node mapping of the pleural space," Chest, vol. 127, no. 5, pp. 1799-1804, 2005.

[122] J. P. Zimmer, S. W. Kim, S. Ohnishi, E. Tanaka, J. V. Frangioni, and M. G. Bawendi, "Size series of small indium arsenidezinc selenide core-shell nanocrystals and their application to in vivo imaging," Journal of the American Chemical Society, vol. 128, no. 8, pp. 2526-2527, 2006.

[123] H. Kobayashi, Y. Hama, Y. Koyama et al., "Simultaneous multicolor imaging of five different lymphatic basins using quantum dots," Nano Letters, vol. 7, no. 6, pp. 1711-1716, 2007.

[124] Y. Hama, Y. Koyama, Y. Urano, P. L. Choyke, and H. Kobayashi, "Simultaneous two-color spectral fluorescence lymphangiography with near infrared quantum dots to map two lymphatic flows from the breast and the upper extremity," Breast Cancer Research and Treatment, vol. 103, no. 1, pp. 23-28, 2007.

[125] S. Kim, Y. T. Lim, E. G. Soltesz et al., "Near-infrared fluorescent type II quantum dots for sentinel lymph node mapping," Nature Biotechnology, vol. 22, no. 1, pp. 93-97, 2004.

[126] Y. T. Lim, S. Kim, A. Nakayama, N. E. Stott, M. G. Bawendi, and J. V. Frangioni, "Selection of quantum dot wavelengths for biomedical assays and imaging," Molecular Imaging, vol. 2, no. 1, pp. 50-64, 2003.

[127] J. D. Smith, G. W. Fisher, A. S. Waggoner, and P. G. Campbell, "The use of quantum dots for analysis of chick CAM vasculature," Microvascular Research, vol. 73, no. 2, pp. 7583, 2007.

[128] D. R. Larson, W. R. Zipfel, R. M. Williams et al., "Watersoluble quantum dots for multiphoton fluorescence imaging in vivo," Science, vol. 300, no. 5624, pp. 1434-1436, 2003.

[129] X. Yu, L. Chen, K. Li et al., "Immunofluorescence detection with quantum dot bioconjugates for hepatoma in vivo," Journal of Biomedical Optics, vol. 12, no. 1, Article ID 014008, 2007.

[130] H. Tada, H. Higuchi, T. M. Wanatabe, and N. Ohuchi, "In vivo real-time tracking of single quantum dots conjugated with monoclonal anti-HER2 antibody in tumors of mice," Cancer Research, vol. 67, no. 3, pp. 1138-1144, 2007.

[131] M. E. Åkerman, W. C. W. Chan, P. Laakkonen, S. N. Bhatia, and E. Ruoslahti, "Nanocrystal targeting in vivo," Proceedings 
of the National Academy of Sciences of the United States of America, vol. 99, no. 20, pp. 12617-12621, 2002.

[132] X. Gao, Y. Cui, R. M. Levenson, L. W. K. Chung, and S. Nie, "In vivo cancer targeting and imaging with semiconductor quantum dots," Nature Biotechnology, vol. 22, no. 8, pp. 969976, 2004.

[133] X. Huang, P. K. Jain, I. H. El-Sayed, and M. A. El-Sayed, "Determination of the minimum temperature required for selective photothermal destruction of cancer cells with the use of immunotargeted gold nanoparticles," Photochemistry and Photobiology, vol. 82, no. 2, pp. 412-417, 2006.

[134] J. Chen, D. Wang, J. Xi et al., "Immuno gold nanocages with tailored optical properties for targeted photothermal destruction of cancer cells," Nano Letters, vol. 7, no. 5, pp. 1318-1322, 2007.

[135] Y. Haba, C. Kojima, A. Harada, T. Ura, H. Horinaka, and K. Kono, "Preparation of poly(ethylene glycol)-modified poly(amido amine) dendrimers encapsulating gold nanoparticles and their heat-generating ability," Langmuir, vol. 23, no. 10, pp. 5243-5246, 2007.

[136] D. K. Kirui, D. A. Rey, and C. A. Batt, "Gold hybrid nanoparticles for targeted phototherapy and cancer imaging," Nanotechnology, vol. 21, no. 10, Article ID 105105, 2010.

[137] X. Huang, I. H. El-Sayed, W. Qian, and M. A. El-Sayed, "Cancer cell imaging and photothermal therapy in the nearinfrared region by using gold nanorods," Journal of the American Chemical Society, vol. 128, no. 6, pp. 2115-2120, 2006.

[138] X. Huang, I. H. El-Sayed, and M. A. El-Sayed, "Applications of gold nanorods for cancer imaging and photothermal therapy," Methods in Molecular Biology, vol. 624, pp. 343-357, 2010.

[139] W. S. Kuo, C. N. Chang, Y. T. Chang et al., "Gold nanorods in photodynamic therapy, as hyperthermia agents, and in nearinfrared optical imaging," Angewandte Chemie - International Edition, vol. 49, no. 15, pp. 2711-2715, 2010.

[140] B. Van De Broek, N. Devoogdt, A. Dhollander et al., "Specific cell targeting with nanobody conjugated branched gold nanoparticles for photothermal therapy," ACS Nano, vol. 5, no. 6, pp. 4319-4328, 2011.

[141] L. R. Hirsch, R. J. Stafford, J. A. Bankson et al., "Nanoshellmediated near-infrared thermal therapy of tumors under magnetic resonance guidance," Proceedings of the National Academy of Sciences of the United States of America, vol. 100, no. 23, pp. 13549-13554, 2003.

[142] C. Loo, L. Hirsch, M. H. Lee et al., "Gold nanoshell bioconjugates for molecular imaging in living cells," Optics Letters, vol. 30, no. 9, pp. 1012-1014, 2005.

[143] C. Loo, A. Lowery, N. Halas, J. West, and R. Drezek, "Immunotargeted nanoshells for integrated cancer imaging and therapy," Nano Letters, vol. 5, no. 4, pp. 709-711, 2005.

[144] X. Huang, P. K. Jain, I. H. El-Sayed, and M. A. ElSayed, "Plasmonic photothermal therapy (PPTT) using gold nanoparticles," Lasers in Medical Science, vol. 23, no. 3, pp. 217-228, 2008.

[145] S. B. Brown and S. H. Ibbotson, "Photodynamic therapy and cancer," BMJ, vol. 339, Article ID b2459, 2009.

[146] J. Cadet, "The photodynamic therapy of cancer cells," Photochemistry and photobiology, vol. 87, no. 1, p. 1, 2011.

[147] A. Lin and S. M. Hahn, "Photodynamic therapy: a light in the darkness?" Clinical Cancer Research, vol. 15, no. 13, pp. 4252-4253, 2009.

[148] M. A. MacCormack, "Photodynamic therapy," Advances in Dermatology, vol. 22, pp. 219-258, 2006.
[149] P. Zhang, W. Steelant, M. Kumar, and M. Scholfield, "Versatile photosensitizers for photodynamic therapy at infrared excitation," Journal of the American Chemical Society, vol. 129, no. 15, pp. 4526-4527, 2007.

[150] B. Ungun, R. K. Prud'homme, S. J. Budijono et al., "Nanofabricated upconversion nanoparticles for photodynamic therapy," Optics Express, vol. 17, no. 1, pp. 80-86, 2009.

[151] D. K. Chatterjee and Z. Yong, "Upconverting nanoparticles as nanotransducers for photodynamic therapy in cancer cells," Nanomedicine, vol. 3, no. 1, pp. 73-82, 2008.

[152] H. S. Qian, H. C. Guo, P. C. L. Ho, R. Mahendran, and Y. Zhang, "Mesoporous-silica-coated up-conversion fluorescent nanoparticles for photodynamic therapy," Small, vol. 5, no. 20, pp. 2285-2290, 2009. 


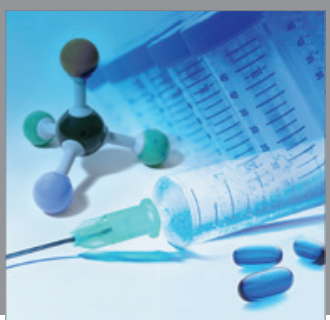

International Journal of

Medicinal Chemistry

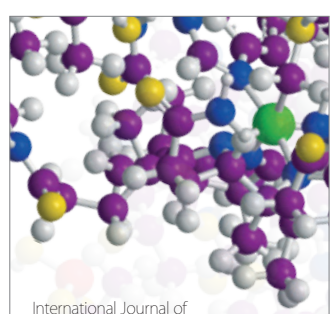

Carbohydrate Chemistry

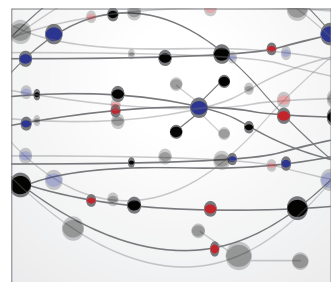

The Scientific World Journal
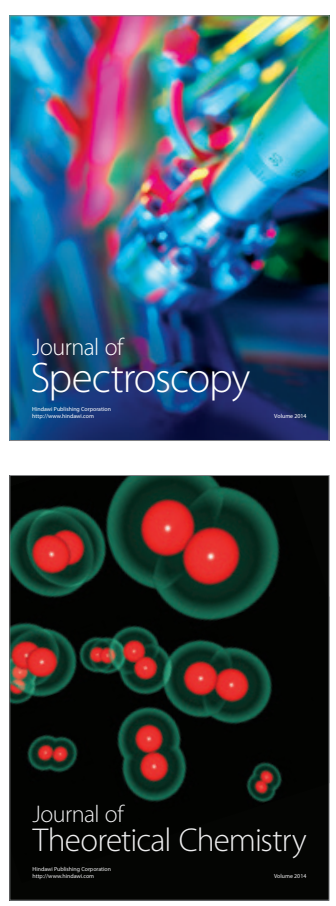
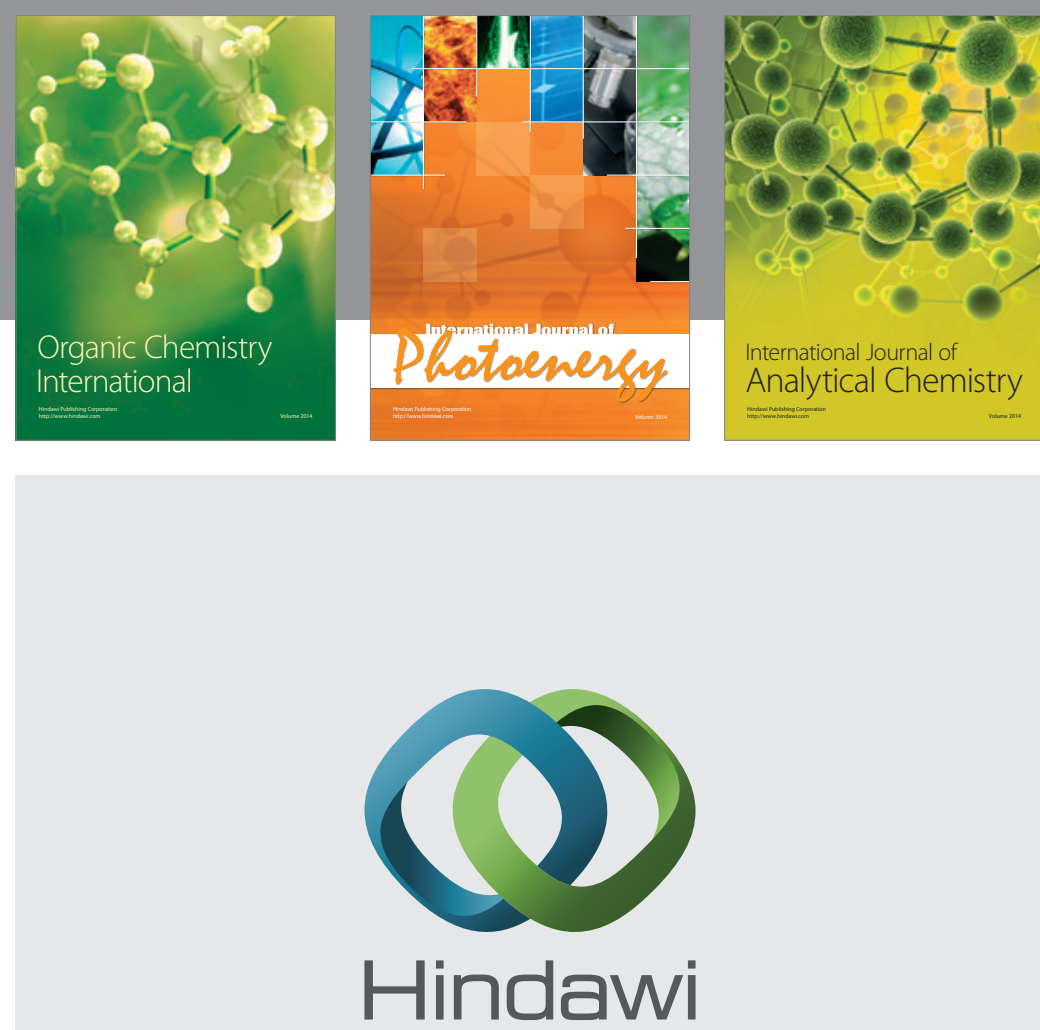

Submit your manuscripts at

http://www.hindawi.com
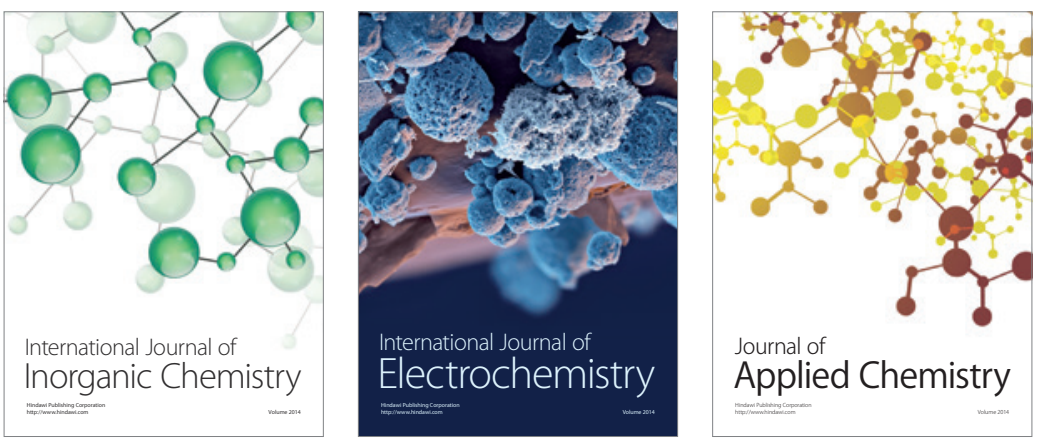

Journal of

Applied Chemistry
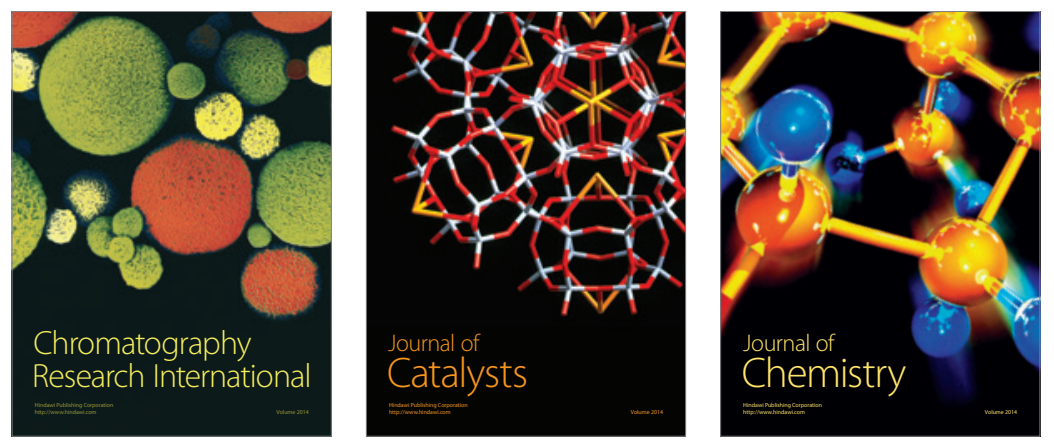
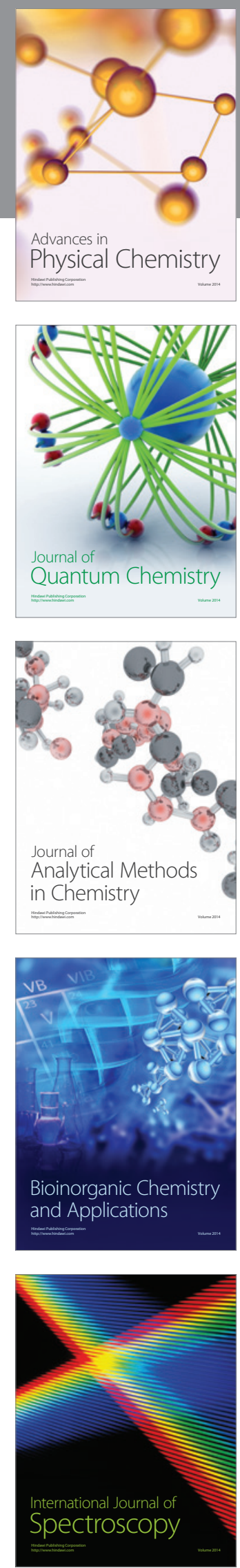